\title{
Correction to: Age-Related Effects of Bariatric Surgery on Early Atherosclerosis and Cardiovascular Risk Reduction
}

Frederik H. W. Jonker ${ }^{1}$ • Vera A. A. van Houten ${ }^{2}$ Leontine H. Wijngaarden ${ }^{1}$.

René A. Klaassen ${ }^{1}$. André A. E. A. de Smet ${ }^{1}$ - André Niezen ${ }^{3}$.

Lodewijk J. D. M. Schelfhout ${ }^{4}$. Tobias A. Bruning ${ }^{5} \cdot$ Erwin van der Harst $^{1}$

Published online: 26 October 2017

(C) Springer Science+Business Media, LLC 2017

\section{Correction to: OBES SURG}

https://doi.org/10.1007/s11695-017-2962-5

Frederik H. W. Jonker and Vera A. A. van Houten contributed equally to this work.

The online version of the original article can be found at https://doi.org/10.1007/s11695-017-2962-5.

Frederik H. W. Jonker

jonkerfrederik@hotmail.com

Vera A. A. van Houten

veravanhouten@gmail.com

Leontine $\mathrm{H}$. Wijngaarden

wijngaardenl@maasstadziekenhuis.nl

René A. Klaassen

klaassenr@maasstadziekenhuis.nl

André Niezen

niezenr@maasstadziekenhuis.nl

Lodewijk J. D. M. Schelfhout

schelfhoutl@maasstadziekenhuis.nl
Tobias A. Bruning

bruningt@maasstadziekenhuis.nl

Erwin van der Harst

harste@maasstadziekenhuis.nl

Department of Surgery, Maasstad Hospital, Maasstadweg 21, 3079 DZ Rotterdam, Netherlands

2 Department of Surgery, Groene Hart Hospital, Gouda, Netherlands

Department of Radiology and Nuclear Medicine, Maasstad Hospital, Rotterdam, Netherlands

4 Department of Internal Medicine, Maasstad Hospital, Rotterdam, Netherlands

5 Department of Cardiology, Maasstad Hospital, Rotterdam, Netherlands 